\title{
Editorial: First Issue on Mean Field Games
}

\author{
Alain Bensoussan $^{1,2}$ - François Delarue ${ }^{3}$
}

Published online: 15 November 2016

(C) Springer Science+Business Media New York 2016

This special issue of the journal Applied Mathematics and Optimization is the first of a two-part volume dedicated to the recent advances on mean field games and, more generally, on large population stochastic control problems. The topic has been knowing a surge of interest since the pioneering works of Lasry and Lions and of Huang, Caines and Malhamé published 10 years ago. There must be several reasons for explaining such a growing interest, among which the following two ones. First, the concept may be applied to various areas, including economy and finance, but also biology or social sciences... Second, the mathematical analysis raises numerous challenging theoretical questions.

Remarkably, the study of mean field games appeal to several topics in mathematics and applied mathematics, such as optimization and game theory, partial differential equations, probability theory, optimal transportation, or scientific computing... Certainly, the fact that the broad of tools involved in the analysis is so wide is another reason why the field has become so popular over the last few years. To wit, the authors who contributed to these two special issues have different backgrounds, some of them being keen on the analytic approach and some others preferring the probabilistic one.

$凶$ François Delarue

delarue@unice.fr

Alain Bensoussan

Alain.bensoussan@utdallas.edu; abensous@cityu.edu.hk

1 The University of Texas at Dallas, 800 West Campbell Road, SM 30 Richardson, TX 75080-3021, USA

2 City University of Hong Kong, 83 Tat Chee Avenue, Kowloon Tong, Hong Kong, China

3 Laboratoire J.A.Dieudonné UMR CNRS-UNS Nº7351 Université de Nice Sophia-Antipolis Parc Valrose, 06108 NICE Cedex 2, France 
Hence, the contributions published in these two issues should reflect the wide variety of techniques which have been developed so far.

This first issue contains eight original articles on mean field games and on mean field control problems, the latter being also known as McKean-Vlasov control problems. Whilst mean field games address the existence of Nash equilibria within in a large population of individuals, mean field control problems are concerned with the optimization of some common reward over a collection of interacting agents obeying a central planner. Although the two problems correspond to different modelings, they are in fact deeply connected. Not only the tools involved in the analysis are pretty much the same, but also there are generic instances of mean field games and of mean field control problems that share the same solutions. For example, so is the case for potential games. In his contribution to this special issue, P. Jameson Graber focuses on this intriguing connection in the framework of linear-quadratic mean field games subject to a common noise.

As for mean field games, several approaches are conceivable to handle mean field optimal control problems. In this issue, Pham and Wei's work, on the one hand, and Buckdahn, Li and Ma's article, on the other hand, investigate two different strategies. In their contribution, H. Pham and X. Wei show, in the discrete time setting for conveniency, how the dynamic programming principle can be applied by enlarging the state space and hence how the apparent time inconsistency of such problems can be bypassed. R. Buckdahn, J. Li and J. Ma focus on the derivation of the Pontryagin principle when the set of controls is non-convex by adapting Peng's seminal spike variation approach. The reader will also find a contribution dedicated to the numerical analysis of this type of problems. Y. Achdou and M. Laurière investigate a numerical method based on an augmented Lagrangian formulation for handling problems with congestion, congestion phenomena intervening in the modeling of crowd motion.

Mean field games with a common noise, as they are presented in the aforementioned Graber's paper, have been receiving more and more attention for the recent years. The terminology "common" refers to the case when players in the game are subject to a common noise, as opposed to the case when players are just subject to independentor idiosyncratic — noises. The word "common" is sometimes replaced by "systemic" to emphasize the fact that the underlying noise affects the whole system and not only a single private player. In their contribution, Y. Achdou, N. Giraud, J.-M. Lasry and P.-L. Lions address a model for mining industry involving production units competing with one another. In this model, the role of the common noise is played by the exogenous demand and the analysis is based upon the introduction of a suitable partial differential equation, which turns out to be an instance of the more general notion of master equation for mean field games.

The master equation, as it appears in the work by Achdou, Giraud, Lasry and Lions, is another quite fascinating concept in the theory of mean field games. Introduced by Lions in his seminal lectures at the Collège de France, it permits to recast the standard formulation of mean field games, based on a forward-backward system of two Kolmogorov-Fokker-Planck and Hamilton-Jacobi-Bellman equations, into a single partial differential equation set on the space of probability measures. It may be especially useful in mean field games with a common noise, as the underlying Kolmogorov-Fokker-Planck and Hamilton-Jacobi-Bellman equations become sto- 
chastic and hence are quite heavy to manipulate. In parametric models, the space over which the master equation is set becomes finite dimensional and the master equation reduces to a standard partial differential equation, which is precisely what happens in Achdou, Giraud, Lasry and Lions' work. However, although the master equation offers a convenient alternative to the forward-backward formulation, the forward-backward system remains of a deep interest, especially when there is no uniqueness to the underlying mean field game. In this regard, the analysis of the forward-backward system associated with deterministic games, also known as first order mean field games, is known to be challenging. In their contribution, D. Gomes, L. Nurbekyan and D. Sedjro address the forward-forward counterpart of the first-order forward-backward mean field game system and discuss the connection between such forward-forward systems and systems of conservation laws. And in their work on a stochastic growthconsumption mean field game, Huang and Nguyen provide a complete solution to the model at hand by solving explicitly the corresponding fixed point forward-backward formulation, which shows, if ever needed, how this latter approach to mean field games remains efficient.

Last, whilst, in most of the papers on mean field games, the analysis is performed for dynamics with values in a continuous state space, the mean field game theory is in fact flexible enough to accommodate dynamics with discrete values. For instance, so is the case in the contribution of Kolokoltsov and Bensoussan. Indeed, V. Kolokoltsov and A. Bensoussan propose a mean field game model for describing the effect of an attack onto a large network of personal computers together with the responses of the computer owners against the cyber-hacker. Differently from other models tackled in this issue, the dynamics are given by jump processes and individual owners may switch from one state to another.

We hope that these two issues on mean field games will supply the reader with a large overview of the recent advances in this very active field. We are especially grateful to all the authors for their contributions. We also warmly thank the editors of the journal for having given us the opportunity to promote the topic. And, last but not least, we thank Pierre-Louis Lions, Jean-Michel Lasry, Peter Caines, Minyi Huang and Roland Malhamé for having initiated this exciting and fascinating subject.

Dallas, Nice, 1st November 2016. 